\title{
Cricoid Ossification Mimicking an Esophageal Foreign Body: A Victim of Overdiagnosis
}

\author{
Rohit Bhardwaj $^{\mathrm{a}} \quad$ Isha Preet Tulia ${ }^{\mathrm{a}}$ Mohamed Shareef PK ${ }^{\mathrm{b}} \quad$ Ankur Gupta \\ Saurabh Makkar ${ }^{\mathrm{a}}$ \\ aDepartment of ENT, Vardhaman Mahaveer Medical College and Safdarjung Hospital, New Delhi, India; \\ bepartment of Otorhinolaryngology, Aster Malabar Institute of Medical Sciences, Kottakkal, India
}

\section{Keywords \\ Aerodigestive tract · Cricoid cartilage ossification . \\ Esophagoscopy · Foreign body · Laryngeal cartilage}

\begin{abstract}
Laryngeal cartilages undergo ossification, usually after the third decade of life. Variations exist in the extent and age of onset of ossification. Radiologically, these ossified cartilages may mimic an aerodigestive tract foreign body. We present an unusual case where the ossified cricoid cartilage masqueraded as a fish bone in the aerodigestive tract. A 48-yearold male patient presented to us with a foreign body sensation and throat pain after consuming fish. We found no fish bone on endoscopic laryngopharyngeal assessment but noted a linear opacity against the C- 6 vertebral body in the aerodigestive tract on a lateral $\mathrm{X}$-ray film of the neck. Rigid esophagoscopy also did not reveal any foreign object, but a computed tomography (CT) scan showed a linear hyperdensity in the wall of the aerodigestive tract, against the C- 6 vertebral body, due to linear ossification in the midline of the cricoid lamina posteriorly. The patient's symptoms improved within 1 week of anti-reflux medications, and he remains asymptomatic. It is possible to encounter such rare cases of
\end{abstract}

karger@karger.com www.karger.com/dmj

Karger"

GOPEN ACCESS
(C) 2021 The Author(s)

Published by S. Karger AG, Basel

This is an Open Access article licensed under the Creative Commons Attribution-NonCommercial-4.0 International License (CC BY-NC) (http://www.karger.com/Services/OpenAccessLicense), applicable to the online version of the article only. Usage and distribution for commercial purposes requires written permission. cartilage ossification while dealing with foreign body aerodigestive tract in adult patients in clinical practice. Proper assessment using CT scan prevents unnecessary procedures and anxiety to the patient.

(c) 2021 The Author(s)

Published by S. Karger AG, Basel

\section{Introduction}

The laryngeal framework is composed of cartilages connected by ligaments and membranes. Ossification of laryngeal cartilages usually begins at the age of 20 years in males and in females at the age of 22 years when skeletal growth is complete [1], and is a degenerative process in hyaline cartilages with increasing age $[1,2]$. Ossification may occur in one or more laryngeal cartilages, although it varies in age of onset and extent. Usually starting first in the thyroid cartilage at about 30 years, it is complete by 65 years of age. Ossification in the cricoid cartilage proceeds from behind forward. Despite being a physiological process, cases of misdiagnosis of ossified laryngeal cartilages as foreign bodies at the upper esophageal sphincter are reported in the literature [1-4], including where laryngeal cartilage ossification mimicked a subglottic polypoidal mass [5]. 


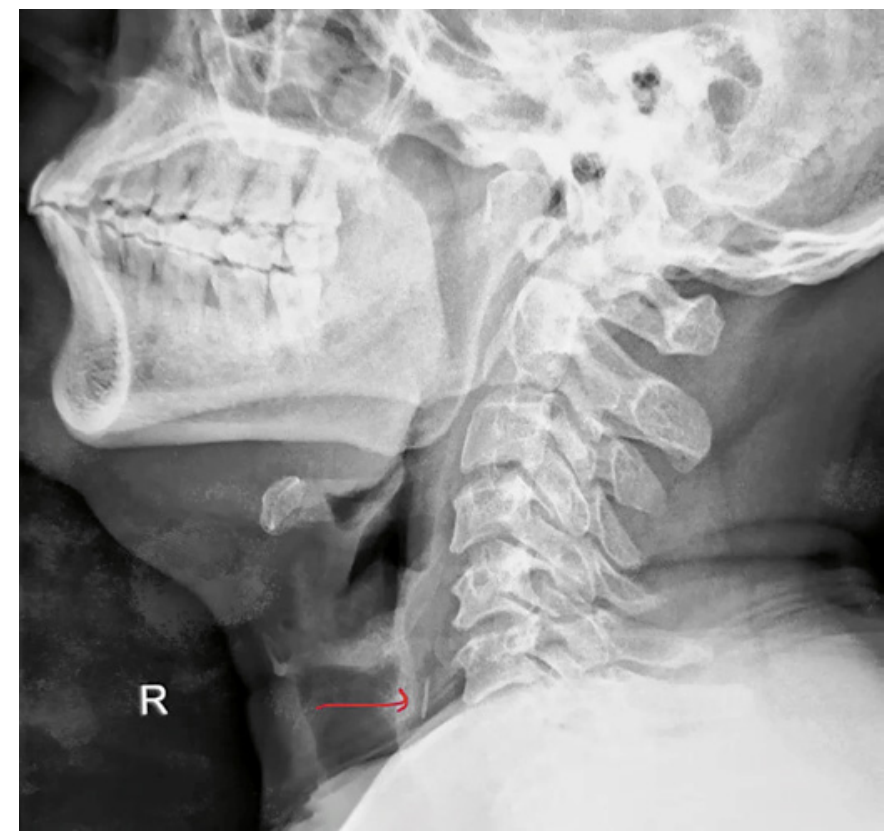

Fig. 1. X-ray lateral film showing vertically placed linear opacity (marked by arrow).

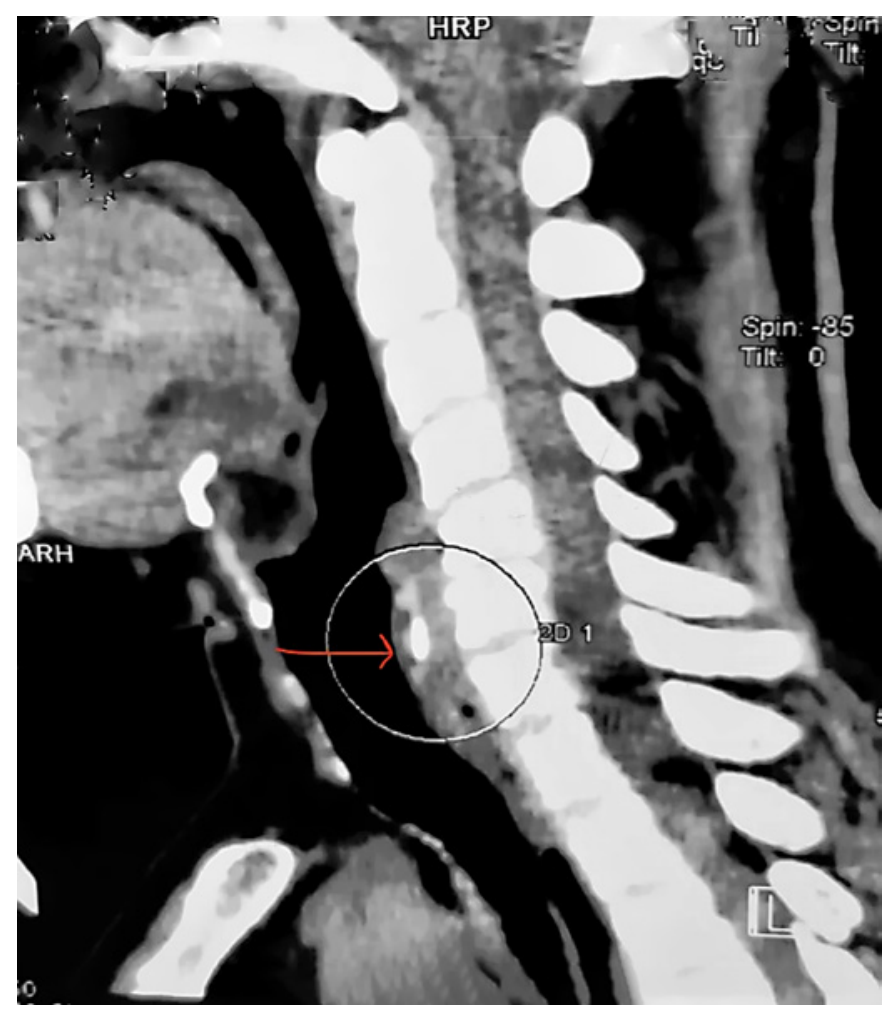

Fig. 2. Sagittal reconstruction CT image showing hyperdensity in the wall of the aerodigestive tract (marked by arrow). CT, computed tomography.
Here, we report a linear ossification of cricoid cartilage mimicking a fish bone in the upper digestive tract. Only few cases of vertical linear ossification of cricoid cartilage lamina posteriorly are reported in the literature $[4,6,7]$, and we report this case due to its rarity and propensity for misdiagnosis and unnecessary explorative surgery like cervical esophagotomy [8].

\section{Case Presentation}

A 48-year-old man presented to the ENT emergency of our hospital on February 2019 with complaints of abnormal pricking sensation and pain in the throat after having fish in his meal the previous night. Endoscopic examination of the laryngopharynx showed features of minimal laryngopharyngeal reflux and no apparent foreign body, that is, fish bone. Palpation of both tonsils, the tonsillolingual sulcus, retromolar trigone, and base of the tongue did not suggest an embedded foreign body. We noted a linear opacity opposite the C-6 vertebral body in the upper aerodigestive tract, on a lateral X-ray film of the neck (shown in Fig. 1). Based on clinical history and radiological assessment, we made a provisional diagnosis of a foreign body (fish bone) in the upper digestive tract at the level of the cricopharyngeal sphincter.

We did not visualize any foreign body, localized trauma, or mucosal ulcer on rigid esophagoscopy under general anesthesia. Since the patient's symptoms persisted, we did a computed tomography (CT) of the neck to exclude an embedded foreign body in the esophageal wall and detect and prevent any ensuing complication.

The CT revealed a dense calcified vertical ridge on the lamina of cricoid cartilage posteriorly. On sagittal reconstruction, the posterior vertically oriented calcification was lying exactly in the region of the posterior lamina (shown in Fig. 2), seen in continuity with the rest of the cricoid rim on axial images (shown in Fig. 3). The vertical length of the density matched the vertical length of the posterior lamina of cricoid cartilage on contiguous sections. The length was also in the range of the reported length of the posterior cricoid lamina of adults. Therefore, what appeared as a foreign body on X-ray soft tissue neck was essentially a calcified posterior cricoid lamina. The patient was reassured regarding the radiological reporting and absence of foreign body. His symptoms improved within 1 week of anti-reflux medications, and he remains asymptomatic after 6 months of follow-up. The patient gave his written informed consent to publish this case and publication of images.

\section{Discussion/Conclusion}

The upper esophageal sphincter (cricopharyngeus) is the usual site where foreign bodies impact after swallowing. Laryngeal cartilages lying in approximation of this sphincter, if ossified, may be erroneously diagnosed as an impacted foreign body. Ossification commonly occurs in one or more laryngeal cartilages as a physiological process 
Fig. 3. Axial CT images at the level of the cricoid ring depicting hyperdensity in the posterior midline of the cricoid lamina (depicted by arrow). CT, computed tomography.

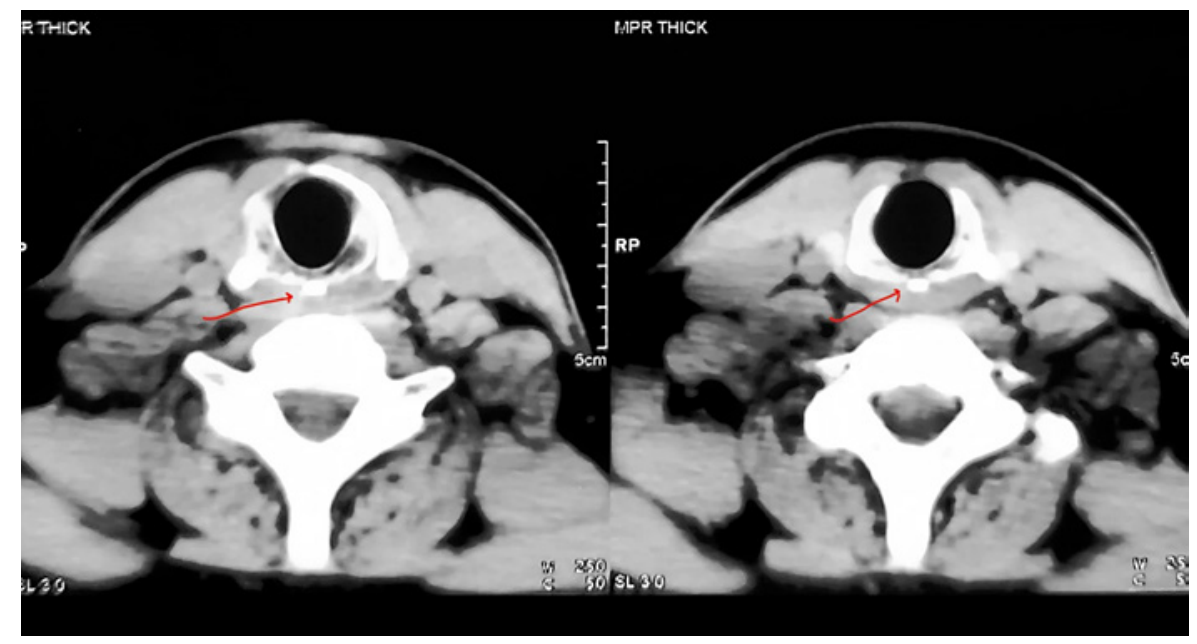

with advancing age, but the age of onset and the extent vary. It usually occurs first in the thyroid cartilage and then in others. Ossification of the cricoid cartilage begins first in the curvilinear superior border of the lamina and then advances from behind forward. Areas of linear ossification may be seen in the lamina posteriorly and oblique superior border of the cricoid arch. The anterior half of the cricoid arch ossifies last. The superior tip of the cricoid lamina and vertical ossification of the posterior margin of the cricoid lamina are most likely to be confused with a foreign body in the aerodigestive tract [1]. The ossification of the cricoid cartilage is seen more in males [2].

The ossification of the laryngeal cartilage follows inconsistent patterns, making differentiation of a pathologic condition from a normal variant in plain radiographs unreliable, most often while distinguishing a partly ossified cartilage from a foreign body. Within the laryngeal complex, the superior margin of the cricoid lamina often ossifies early, before the remainder of the signet portion of the cricoid. This linear ossification is often mistaken for a foreign body [2]. The vertical ossification of the posterior margin of the cricoid lamina may mimic a bone, as was seen in the case described.

The clinician should be aware of these ossification patterns and radiological appearance of the cricoid cartilage in order to avoid misdiagnosis of these conditions as an impacted foreign body. To differentiate the ossification of the cricoid cartilage from an impacted foreign body, emphasis has to be given to the extent of ossification. The physiological ossification never goes beyond the upper and lower cricoid borders, whereas opacification of an impacted foreign body commonly crosses these limits [3]. The European Society of Gastrointestinal Endoscopy (ESGE) guidelines on removal of foreign bodies in the upper gastrointestinal tract in adults recommend plain X-ray if ingestion of radiopaque objects is suspected or type of object is unknown, and a CT scan for those patients with suspected perforation or other complications that may require surgery [9]. We believe additionally a CT scan may be recommended for doubtful bony foreign bodies. On ultrasonography, all calcified objects cast an acoustic shadow posteriorly, while on MRI they appear hypointense, thus merging with air shadows, making differentiation difficult. Therefore, a CT scan is a superior modality to confirm the diagnosis in such cases.

In our case, the vertical linear opacification between the upper and lower borders of the cricoid lamina posteriorly was misdiagnosed as an impacted foreign body in the hypopharynx. We suspected an abnormal calcification of the cricoid cartilage because the opacification appeared contiguous with the cricoid rim on axial section, in the setting of a minimally symptomatic patient. This is demonstrated on sagittal reconstruction, where the density was seen in continuity with the posterior lamina of the cricoid cartilage and was within its confines anatomically. CT scan is used to distinguish normal ossification patterns in laryngeal cartilages from pathologies, particularly in carcinoma larynx [10]. We propose, similarly, it should be the modality of investigation in confusing cases of aerodigestive foreign bodies as well.

To conclude, based on learning offered by this case, relying on CT scan in doubtful radiographic findings of laryngeal cartilage ossification, such as in our case of linear opacity at the cricopharyngeal level, can rule out a 
foreign body. This will help avoid the patient suffering, as well as the unnecessary burden of repeated diagnostic and invasive interventional attempts on the health-care system.

\section{Statement of Ethics}

The patient gave his written informed consent to publish this case and publication of images.

\section{Conflict of Interest Statement}

The authors have no conflicts of interest to declare.

\section{Funding Sources}

The authors did not receive any funding.

\section{Author Contributions}

R.B., I.P.T., M.S.P.K., A.G., and S.M.: conception of the work; acquisition, analysis, and interpretation of data for the work; drafting and revising the manuscript; final approval of the version to be published; and agreement to be accountable for all aspects of the work in ensuring that questions related to the accuracy or integrity of any part of the work are appropriately investigated and resolved. I.P.T.: as corresponding author, primary responsibility for communication with the journal during manuscript submission, peer review, and the publication process.

\section{References}

1 Hately W, Evison G, Samuel E. The pattern of ossification in the laryngeal cartilages: a radiological study. Br J Radiol. 1965;38(452): 585-91.

2 Mupparapu M, Vuppalapati A. Ossification of laryngeal cartilages on lateral cephalometric radiographs. Angle Orthod. 2005;75(2): 196-201.

3 O'Bannon RP, Grunow OH. The larynx and pharynx radiologically considered. South Med J. 1954;47:310-6.

4 Richardson GS, Albuquerque NM. Unusual calcification of cricoid cartilage masquerading as foreign body in esophagus. AMA Arch Otolaryngol. 1955;62(3):316-8.
5 Kim NR, Kim DY, Hwang HY, Chung DH. A case of ossified laryngeal cartilage mimicking a subglottic mass. Am J Otolaryngol. 2012; 33(2):282-4.

6 Wakisaka N, Miwa T, Yoshizaki T, Furukawa M. Cricoid ossification mimicking an impacted foreign body. J Laryngol Otol. 2006 Jul; 120(7):E24.

$7 \mathrm{Ng}$ SJK, Lee JKT, Thian YL. Cricoid ridge ossification mimicking ingested fish bone on plain radiography: prevalence in Singapore. Singapore Med J. 2018;59(7):356-9.
8 Cianci P, Tartaglia N, Altamura A, Di Lascia A, Fersini A, Neri V, et al. Cervical esophagotomy for foreign body extraction: a case report and extensive literature review of the last 20 years. Am J Case Rep. 2018;19:400-5.

9 Birk M, Bauerfeind P, Deprez PH, Häfner M, Hartmann D, Hassan C, et al. Removal of foreign bodies in the upper gastrointestinal tract in adults: European society of gastrointestinal endoscopy (ESGE) clinical guideline. Endoscopy. 2016;48(5):489-96.

10 Adolphs AP, Boersma NA, Diemel BD, Eding JE, Flokstra FE, Wegner I, et al. A systematic review of computed tomography detection of cartilage invasion in laryngeal carcinoma. Laryngoscope. 2015;125(7):1650-5. 